\title{
Spatial Analysis of Tourist Spots in Central Tokyo Using Online Geotagged Photographs from Flickr
}

\author{
Bochra Bettaieb $^{\mathrm{a},} *$, Yoshiki Wakabayashi ${ }^{\mathrm{b}}$ \\ ${ }^{a}$ Graduate Student, Department of Geography, Tokyo Metropolitan University, bochra_bettaieb@hotmail.fr \\ ${ }^{b}$ Department of Geography, Tokyo Metropolitan University, wakaba@tmu.ac.jp \\ * Corresponding author
}

Keywords: photo-sharing service, big data, hot spot analysis, Tokyo

\begin{abstract}
:
Advancements in information and communication technologies enable us to trace human activities through digital media; networks of fixed or mobile sensors, such as smartphones, global positioning systems, and travel cards, can monitor the timing and locations of human behavior throughout the day. These new sources of geospatial data provide the possibility of analyzing high-resolution big data to capture patterns of human spatial behavior across space and time. Online photo-sharing services are comprised of such sources. This study analyzes the geocoded photos from a photosharing service to make a comparison of the varying spatial patterns of tourist attractions in Tokyo as differentiated between those taken by Japanese and foreign visitors.

We selected three places (Shinjuku, Asakusa, and Ginza) as study districts from the five top places frequently visited by foreigners based on the 2014 Survey Report of Foreigner Behavior. The data were downloaded from Flickr, one of the most popular online platforms for people to share their travel experiences by uploading photos. We used the data of geocoded photos from 2014, taken within $1 \mathrm{~km}$ from Shinjuku, Ginza, and Asakusa stations. Information on the nationality of contributors recorded in the Flickr data was used to compare the tourist spots of Japanese and foreign visitors. To identify the tourist spot, we employed a hotspot analysis using geographic information systems.

The obtained results showed some tourist spots common to Japanese and foreign visitors in three study areas, namely, places close to railroad stations. However, the distribution of foreigners' photographs showed a higher spatial concentration than those taken by Japanese tourists. This tendency is consistent with the findings obtained by Suzuki and Wakabayashi (2008), wherein they compared photos of tourist attractions in guide books of Tokyo. This comparative difference can be explained by the accessibility of railroad stations; in other words, foreigners rely more on public transportation during their comparatively short stays.

Spatial patterns of tourist spots varied with the study district in question. In Shinjuku and Ginza, there are differences in tourist spots between Asians and Europeans. Particularly, European tourists' hot spots tend to be more scattered than Asians. However, such differences are not observed in Asakusa, where tourists' hot spots are concentrated around Sensoji Temple and Tokyo Sky Tree. The reason for this is that tourist attractions are concentrated in and around specific facilities in the Asakusa district. Hence, the variations of tourist spots obtained by analyzing the geotagged photographs on Flickr can be explained by the difference in behavior and interest of foreign tourists between countries, as well as the spatial distribution of tourist attractions.
\end{abstract}

